ARTICLE

DOI: $10.1038 / s 41467-017-00048-y$

\title{
Cascaded exciton energy transfer in a monolayer semiconductor lateral heterostructure assisted by surface plasmon polariton
}

Jinwei Shi ${ }^{1,2,3}$, Meng-Hsien Lin', I-Tung Chen ${ }^{4}$, Nasim Mohammadi Estakhri ${ }^{5,6}$, Xin-Quan Zhang ${ }^{4}$, Yanrong Wang ${ }^{2}$, Hung-Ying Chen ${ }^{1}$, Chun-An Chen ${ }^{4}$, Chih-Kang Shih ${ }^{3}$, Andrea Alù (1) ${ }^{6}$, Xiaoqin Li ${ }^{3}$, Yi-Hsien Lee ${ }^{4} \&$ Shangjr Gwo ${ }^{1,7}$

Atomically thin lateral heterostructures based on transition metal dichalcogenides have recently been demonstrated. In monolayer transition metal dichalcogenides, exciton energy transfer is typically limited to a short range $(\sim 1 \mu \mathrm{m})$, and additional losses may be incurred at the interfacial regions of a lateral heterostructure. To overcome these challenges, here we experimentally implement a planar metal-oxide-semiconductor structure by placing a $\mathrm{WS}_{2} / \mathrm{MoS}_{2}$ monolayer heterostructure on top of an $\mathrm{Al}_{2} \mathrm{O}_{3}$-capped $\mathrm{Ag}$ single-crystalline plate. We find that the exciton energy transfer range can be extended to tens of microns in the hybrid structure mediated by an exciton-surface plasmon polariton-exciton conversion mechanism, allowing cascaded exciton energy transfer from one transition metal dichalcogenides region supporting high-energy exciton resonance to a different transition metal dichalcogenides region in the lateral heterostructure with low-energy exciton resonance. The realized planar hybrid structure combines two-dimensional light-emitting materials with planar plasmonic waveguides and offers great potential for developing integrated photonic and plasmonic devices.

\footnotetext{
${ }^{1}$ Department of Physics, National Tsing-Hua University, Hsinchu 30013, Taiwan. ${ }^{2}$ Department of Physics and Applied Optics Beijing Area Major Laboratory, Beijing Normal University, Beijing 100875, China. ${ }^{3}$ Department of Physics, The University of Texas at Austin, Austin, Texas 78712, USA. ${ }^{4}$ Department of Materials Science and Engineering, National Tsing-Hua University, Hsinchu 30013, Taiwan. ${ }^{5}$ Department of Electrical and Systems Engineering, University of Pennsylvania, Philadelphia, Pennsylvania 19104, USA. ${ }^{6}$ Department of Electrical and Computer Engineering, The University of Texas at Austin, Austin, Texas 78712, USA. ${ }^{7}$ National Synchrotron Radiation Research Center, Hsinchu 30076, Taiwan. Correspondence and requests for materials should be addressed to X.L. (email: elaineli@physics.utexas.edu) or to Y.-H.L. (email: yhlee.mse@mx.nthu.edu.tw) or to S.G. (email: gwo@phys.nthu.edu.tw)
} 
M aterials in the family of transition metal dichalcogenides (TMDs) form planar honeycomb lattices with closely matched lattice constants, making it possible to grow monolayer-thick lateral heterostructures (LHSs) using chemical vapor deposition $(\mathrm{CVD})^{1-5}$. Consequently, novel device applications can be envisioned based on LHSs or lateral superlattices consisting of adjacent regions of materials with dissimilar band gaps. Following interband excitations, excitons (electron-hole pairs) with exceptionally large binding energy (e.g., for $\mathrm{WS}_{2}, 320 \mathrm{meV}^{6}$ are formed, making them stable even at room temperature and are relevant for device applications. For device applications based on two-dimensional (2D) TMDs, excitons created via local optical or electrical excitation in one TMD material needs to be transferred to different TMD regions in the LHS to activate the entire active material. Cascaded exciton energy transfer in LHS from one TMD region to another could enable flexible devices, taking advantage of the versatile lateral band structure engineering. Such a cascaded exciton energy transfer process is critical for optoelectronic devices (e.g., displays $^{7}$, photovoltaics ${ }^{8}$ and cascaded fluorescence cameras ${ }^{9}$ ), which are usually based on a variety of materials such as multicolour colloidal quantum dots and polymer films. However, several challenges exist in effective exciton energy transfer in TMDs. Importantly, exciton diffusion is typically limited to a rather short range $(\sim 1 \mu \mathrm{m})$ due to ultrafast exciton recombination lifetime and moderate exciton mobility in TMDs ${ }^{10-14}$. Furthermore, one dimensional (1D) confinement potential may form at the heterojunction of an LHS due to band bending $3,15,16$, causing exciton trapping and additional energy loss at the interface.

Here, we adopt a 2D metal-oxide-semiconductor (MOS) hybrid material platform to address the challenges of long-range exciton energy transfer on a TMD LHS. Our MOS structure consists of a $\mathrm{WS}_{2} / \mathrm{MoS}_{2}$ LHS transferred to a single-crystalline plasmonic plate (Ag plate) covered with a few nanometer thick $\mathrm{Al}_{2} \mathrm{O}_{3}$ layer ${ }^{17}$. The large optical density of states in the plasmonic structures allows the excitonic energy to be effectively transferred to surface plasmon polaritons (SPPs), which can propagate more than $100 \mu \mathrm{m}$ in the visible frequency range ${ }^{17}$, facilitating longrange exciton energy transfer in TMD LHS. Specifically, we demonstrate that this planar MOS structure supports isotropic energy transfer with a propagation length $\sim 40 \mu \mathrm{m}$, which is two orders of magnitude longer than the exciton diffusion length in bare TMD monolayers. Despite the large absorption coefficient of monolayer TMD at the exciton resonance, this propagation length is only about a factor of two shorter than the SPP propagation distance without the TMD layer, as confirmed by simulations. Furthermore, we show that the energy loss at the interface can be minimized in high-quality LHSs with atomically sharp interfaces. Our work suggests that a hybrid photonic/ plasmonic material platform can leverage the advantages of both components. The TMD layers act as efficient light absorbers and emitters due to the concentrated, large oscillator strength associated with the exciton resonance ${ }^{18}$. However, the ultrafast exciton recombination dynamics ${ }^{12-14}$ seriously restrict the exciton energy transfer distance. Here, this limitation is circumvented by coupling the TMD layer to a low-loss plasmonic substrate, which supports waveguiding and energy transfer via SPPs over a sufficiently long distance and offers additional benefits such as the capability of shaping and enhancing the near field ${ }^{19-21}$. In contrast to the previously reported exciton-SPP or SPP-exciton conversion in $1 \mathrm{D}$ hybrid structures consisting of $\mathrm{Ag}$ nanowires and monolayer $\mathrm{TMDs}^{22}{ }^{23}$, the demonstrated long-distance exciton energy transfer process contains a complete cascaded exciton-SPP-exciton conversion in a fully planar geometry. This hybrid material platform offers promising perspectives in future integrated photonic/plasmonic applications by merging plasmonics with atomically thin semiconductors and their unique heterostructures ${ }^{24-26}$.

\section{Results}

Exciton energy transfer assisted by SPP. In Fig. 1a, we show a conceptual schematic of the energy transfer process in the MOS structure. An above-bandgap laser excitation ( 405 or $532 \mathrm{~nm}$ ) is normally incident on the wide-gap $\mathrm{WS}_{2}$ region and creates excitons with photon emission energy near $\sim 630 \mathrm{~nm}$, as illustrated in Fig. 1b. The excitons then excite SPPs in the Ag plate via near-field, non-radiative energy transfer. The natural 2D geometric match between the atomically thin LHS and the plasmonic plate confines the excitonic energy in the 2D MOS structure and allows energy carried by SPPs to propagate on the planar waveguide. In the narrow-gap $\mathrm{MoS}_{2}$ region, the SPP is out-coupled at specific sites,which are created through intentionally introduced disruptions to the $2 \mathrm{D}$ structure, e.g., slots created via atomic force microscope (AFM) lithography or $\mathrm{SiO}_{2}$ spheres. The near-field of SPPs at the out-coupled site then locally excites $\mathrm{MoS}_{2}$ excitons $(\sim 670 \mathrm{~nm})$, completing the exciton-SPP-exciton conversion process. The energy transfer occurs in a cascaded fashion involving energy transfer from a wide-gap region to a narrow-gap region in the LHS.

In this work, the material quality of the planar MOS photonic/plasmonic material platform is crucial for the successful demonstration of long-distance energy transfer. The TMD LHS typically consists of two concentric triangular regions. Depending on the particular CVD procedures used, the $\mathrm{WS}_{2} / \mathrm{MoS}_{2}$ interface conditions can be drastically different (see Methods for details). A one-step growth procedure ${ }^{1}$ produces as-synthesized $\mathrm{WS}_{2} / \mathrm{MoS}_{2} \mathrm{LHS}$ with inner triangles comprising of $\mathrm{MoS}_{2}$ The heterojunction of this $\mathrm{WS}_{2} / \mathrm{MoS}_{2}$ LHS displays an ideal 1D interface along the zigzag direction with an atomically sharp interface without compositional fluctuations or alloyed phases, as shown in the scanning transmission electron microscopy (STEM) image (Fig. 1c). This high-quality LHS enables energy transfer across the interface without measurable loss at the interfacial region. In contrast, a two-step growth procedure yields LHS (inner triangle: $\mathrm{WS}_{2}$ ) with rough interfacial regions decorated with stacked layers of small $\mathrm{MoS}_{2}$ flakes, as shown in Supplementary Fig. 1 and Note 1. The significant refractiveindex change at the interface of the LHS leads to a visible energy loss, as demonstrated below. The Ag plates were grown using a room temperature wet-chemical synthesis method recently developed by us ${ }^{17}$. A few nanometer thick, atomically smooth $\mathrm{Al}_{2} \mathrm{O}_{3}$ layer was immediately grown by using the atomic layer deposition (ALD) technique on top of the Ag plate after removal from the colloidal solution. This oxide layer not only prevents the Ag plate from oxidation/degradation in the ambient environment, but also creates a dielectric spacer to reduce possible photoluminescence (PL) quenching resulting from non-radiative energy transfer between the TMD monolayer and the Ag plate.

First, we examined the excitonic energy transfer process in an LHS with a rough interface, as shown in the inset of Fig. 2a. Excitons optically created in the inner triangular $\mathrm{WS}_{2}$ region act as exciton point sources and excite the SPP modes bound to the MOS structure. The excited SPPs propagate away from the launching site isotropically and are out-coupled at all triangular interfaces due to the significant change in effective refractive index in the rough interfacial region. This process is visually demonstrated in Fig. 2a. A spatial map of the PL signal from the entire TMD heterostructure was collected (see Methods) while the excitation laser was fixed at the centre of the $\mathrm{WS}_{2}$ triangle. The PL spectra collected from both the launching site (red curve) and collection site (blue curve) near the interface of the LHS are 


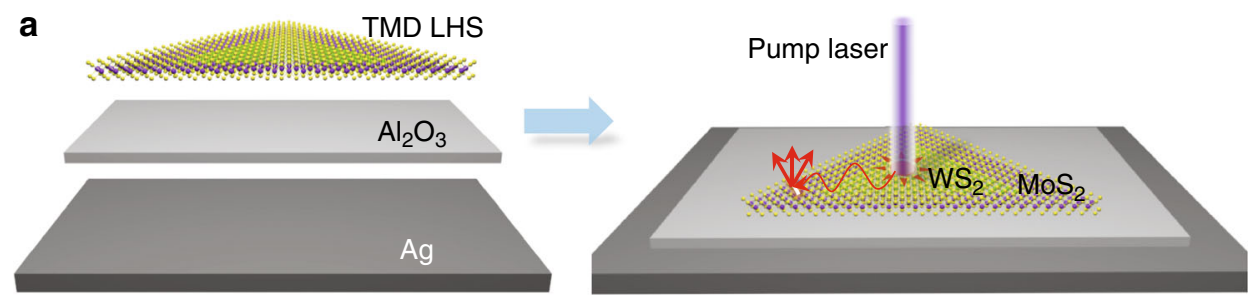

b
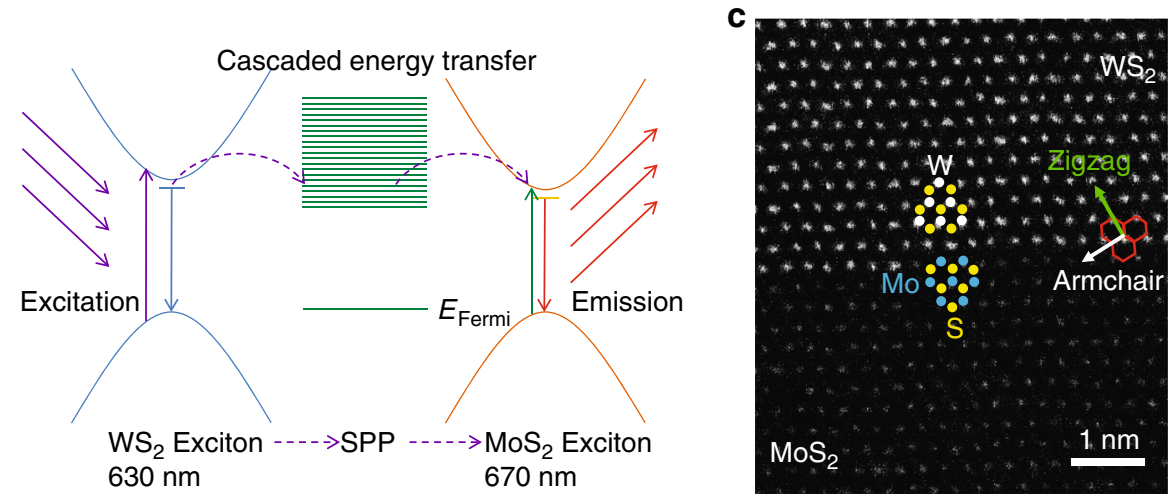

Fig. 1 Schematic illustrations of the metal-oxide-semiconductor (MOS) structure and the cascaded exciton energy transfer process assisted by surface plasmon polariton. a A colloidal $\mathrm{Ag}$ plate is coated with $\sim 3 \mathrm{~nm} \mathrm{Al}_{2} \mathrm{O}_{3}$ immediately after it is taken out of the solution. The as-grown monolayer-thick $\mathrm{WS}_{2} / \mathrm{MoS}_{2}$ lateral heterostructure (LHS) is transferred to the $\mathrm{Ag}$ plate using a polymethyl methacrylate (PMMA)-assisted transfer technique. An excitation laser is normally incident on the $\mathrm{WS}_{2}$ region, and the photoluminescence $(\mathrm{PL})$ from the interface or a specified $M o S_{2}$ region is detected. $\mathbf{b}$ Energy transfer diagram describing the exciton-SPP-exciton conversion process. c High-resolution annular dark-field scanning transmission electron microscopy (STEM) image of a monolayer LHS grown with an atomically sharp interface

a
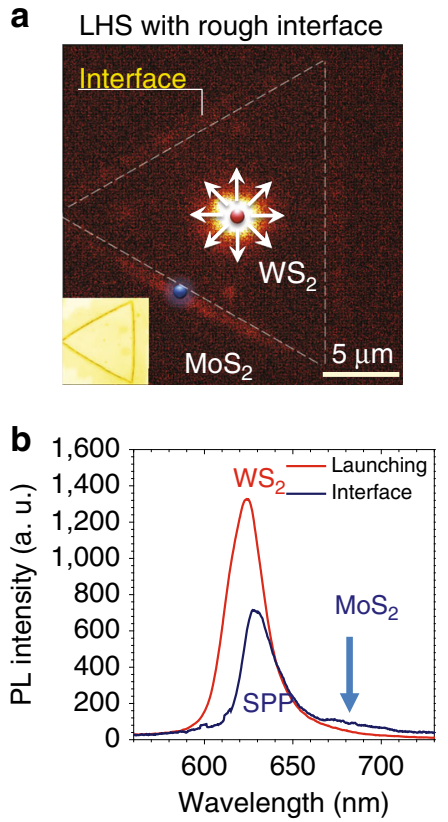

C LHS with smooth interface
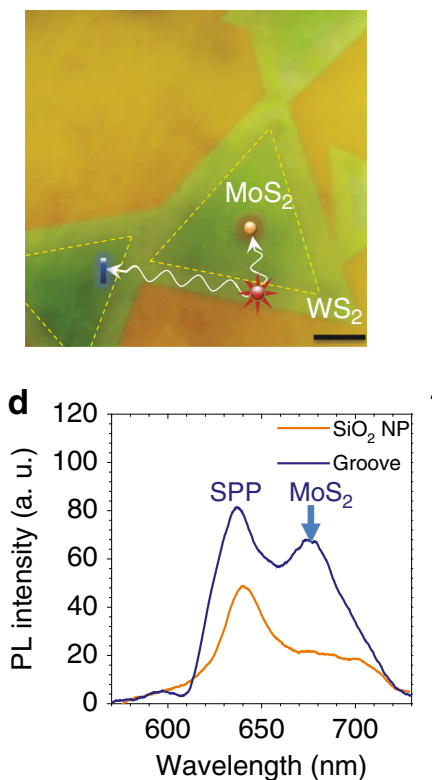

e
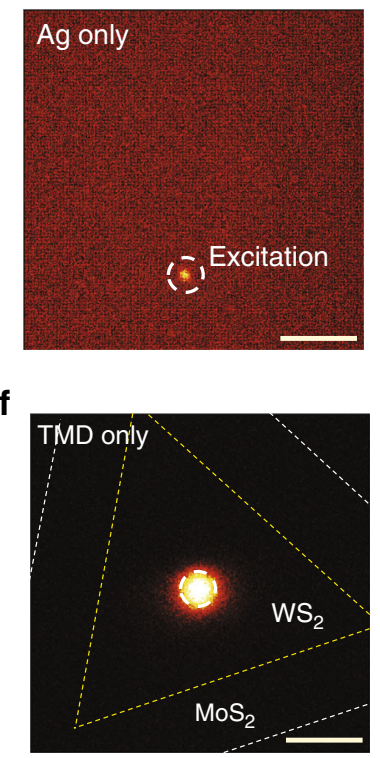

Fig. 2 Experimental demonstration of SPP-assisted exciton energy transfer in a $\mathrm{WS}_{2} / \mathrm{MoS}_{2} \mathrm{TMD} \mathrm{LHS}$. a PL spatial mapping from a metal-oxidesemiconductor (MOS) structure in which the LHS has rough interfaces. Excitons in $\mathrm{WS}_{2}$ (indicated by the red dot) created in the centre of the inner triangle are converted to SPPs, which propagate isotropically and are out-coupled at the interface, exciting the excitons from the MoS $_{2}$ region (indicated by the blue dot). $\mathbf{b}$ PL spectra collected at the launching site and at the interface of the LHS shown in a. c An optical microscopy image of monolayer thick MoS 2 / $W_{2}$ LHS with atomically sharp interfaces. The laser excitation site (indicated by the red star), the out-coupling sites at a $\mathrm{SiO}_{2}$ nanosphere (indicated by the orange dot), and a groove on the $\mathrm{Ag}$ plate (indicated by the blue slit) are labeled on the image. $\mathbf{d}$ The spectra taken at the out-coupling sites of the nanosphere and the groove, respectively. e, $\mathbf{f}$ The $\mathrm{PL}$ spatial mapping images obtained from an $\mathrm{Ag}$ plate alone $\mathbf{e}$, and from a TMD LHS on a $\mathrm{Si} / \mathrm{SiO}{ }_{2}$ substrate $\mathbf{f}$. The scale bars in all figures correspond to $5 \mu \mathrm{m}$ 

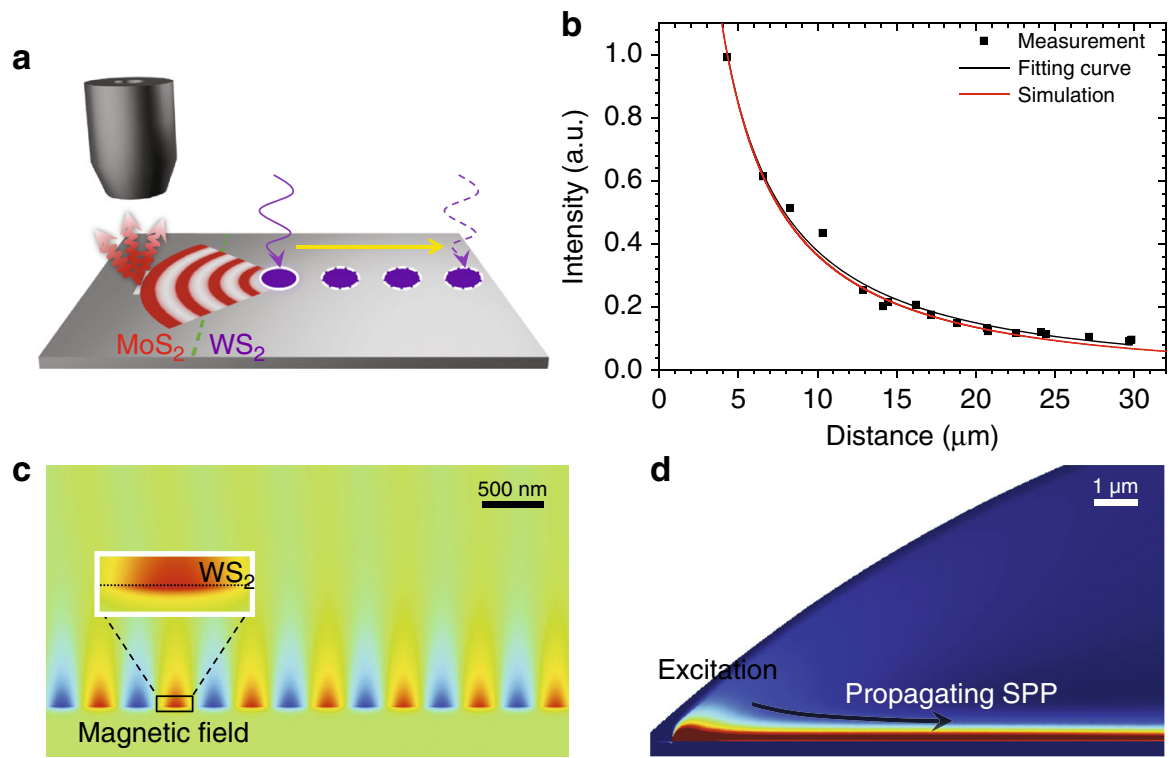

Fig. 3 Measurements and simulations of exciton energy propagation process. a Schematic of measurement geometry for determining the exciton energy propagation length. The PL intensity is collected at a fixed location on the $\mathrm{MoS}_{2}$ region, beneath which a groove is fabricated on the $\mathrm{Ag}$ plate. The excitation laser spot is moved away from the groove during measurements. b Measured PL intensity (black points) vs. increasing distance between the collection and launching sites. Two curves correspond to a fitting result (black curve) and the simulated energy decay behaviour in the MOS structure (red curve). c Simulated near-field profile suggests that a significant percentage of the transferred energy is distributed outside of the TMD LHS monolayer. d Simulated power-density profile and the decay behavior along the propagation direction

shown in Fig. 2b. In the spectrum collected from the rough interfacial region, the resonance near $\sim 630 \mathrm{~nm}$ displays a small red shift in comparison to the spectrum collected at the launching site. This peak likely arises from directly scattered SPPs, which exhibit lower loss at longer wavelength. A weaker peak near 670 $\mathrm{nm}$ (indicated by the blue arrow) is observed in the spectra at collection sites near the interface. This peak coincides well with the excitonic energy in $\mathrm{MoS}_{2}$. This experiment demonstrates the possibility of cascaded excitonic energy transfer assisted by SPPs in TMD-based hybrid materials.

We then investigated the LHS with an atomically sharp interface. In this case, following the exciton-SPP excitation in the $\mathrm{WS}_{2}$ region at the outer triangle, the SPPs propagate through the interface region with little scattering loss because of the small difference in the refractive indices between two TMD regions. In order to observe the second step in the energy conversion process (i.e., SPP to exciton), either a nanogroove patterned by AFM lithography or a $\mathrm{SiO}_{2}$ nanosphere of $500 \mathrm{~nm}$ diameter placed at the selected out-coupling site by using AFM manipulation (illustrated in Fig. 2c) was utilized. Both methods out-couple SPPs, which can also excite the excitons in $\mathrm{MoS}_{2}$ either due to translational symmetry breaking or local hot SPP spots. Therefore, the spectra at the collection sites (orange dot and blue groove) feature a resonance near $630 \mathrm{~nm}$ dominated by scattered SPPs excited by excitons in the $\mathrm{WS}_{2}$ region and the exciton resonance in $\mathrm{MoS}_{2}$ near $670 \mathrm{~nm}$ excited by the out-coupled SPPs, as shown in Fig. 2d. These experimental results demonstrate that SPP-assisted exciton energy transfer from one to the other region of the TMD LHS occurs with a minimal scattering loss at the atomically sharp interface.

To confirm that the exciton-SPP-exciton conversion process is indeed responsible for the observed phenomena, we performed two control experiments. First, the excitation laser was moved to a region of the Ag plate without any TMDs. Neither propagating SPP nor PL signal was detected, as shown in Fig. 2e. These experiments confirm the high surface quality of Ag plate; incident plane waves cannot excite SPP modes on a planar metal/dielectric interface due to the momentum mismatch while SPP modes on a rough metallic surface can be directly excited. In other words, optical excitation in the $\mathrm{WS}_{2}$ region indeed induces the point sources necessary to launch the SPPs in the investigated MOS hybrid platform. We then mapped PL from a TMD heterostructure on a $\mathrm{Si} / \mathrm{SiO}_{2}$ substrate with a fixed excitation location, as shown in Fig. 2f. No PL signal from other regions of the sample was collected except for at the excitation spot, indicating that the exciton diffusion length is within the spot size. This experiment demonstrates that long-distance energy transfer can only be realized in the hybrid MOS platform.

Energy transfer distance. Next, we quantify the propagation length associated with the SPP-assisted exciton energy transfer process. A series of PL spectra have been collected at the same position around the out-coupling groove while the excitation laser spot was moved away from the collection spot (Fig. 3a). In Fig. $3 b$, the integrated PL intensity is plotted as a function of the distance between the launching site on $\mathrm{WS}_{2}$ and the collection site on $\mathrm{MoS}_{2}$. Full-wave simulations (see Methods for details), employing experimentally measured optical constants of crystalline $\mathrm{Ag}^{27}$ and $\mathrm{WS}_{2}{ }^{28}$, predict $\sim 34 \mu \mathrm{m}$ decay length in the presence of the $\mathrm{WS}_{2}$ layer, as shown with solid redline in Fig. 3b. The magnetic field (snapshot in time) and power-density distribution of the SPP wave are shown in Figs. 3c, d. Noticeably, the SPP wave is significantly extended in air (upper region of the surface waveguide), resulting in large propagation distance of the mode, in spite of the moderately large absorption in $\mathrm{WS}_{2}$ and $\mathrm{Ag}$. The propagation distance also strongly depends on the distance between the absorbing $\mathrm{WS}_{2}$ layer to the Ag and oxide interface, where the field intensity is maximal. In the present MOS structure, the $\mathrm{WS}_{2}$ is very close to the metal-dielectric interface $(\sim 3 \mathrm{~nm})$. Thus, a very thin layer of $\mathrm{WS}_{2}$ reduces the propagation distance by a factor of three from $\sim 100 \mu \mathrm{m}$ in the bare plasmonic waveguide to $\sim 34 \mu \mathrm{m}$ in the MOS structure. The measured data 


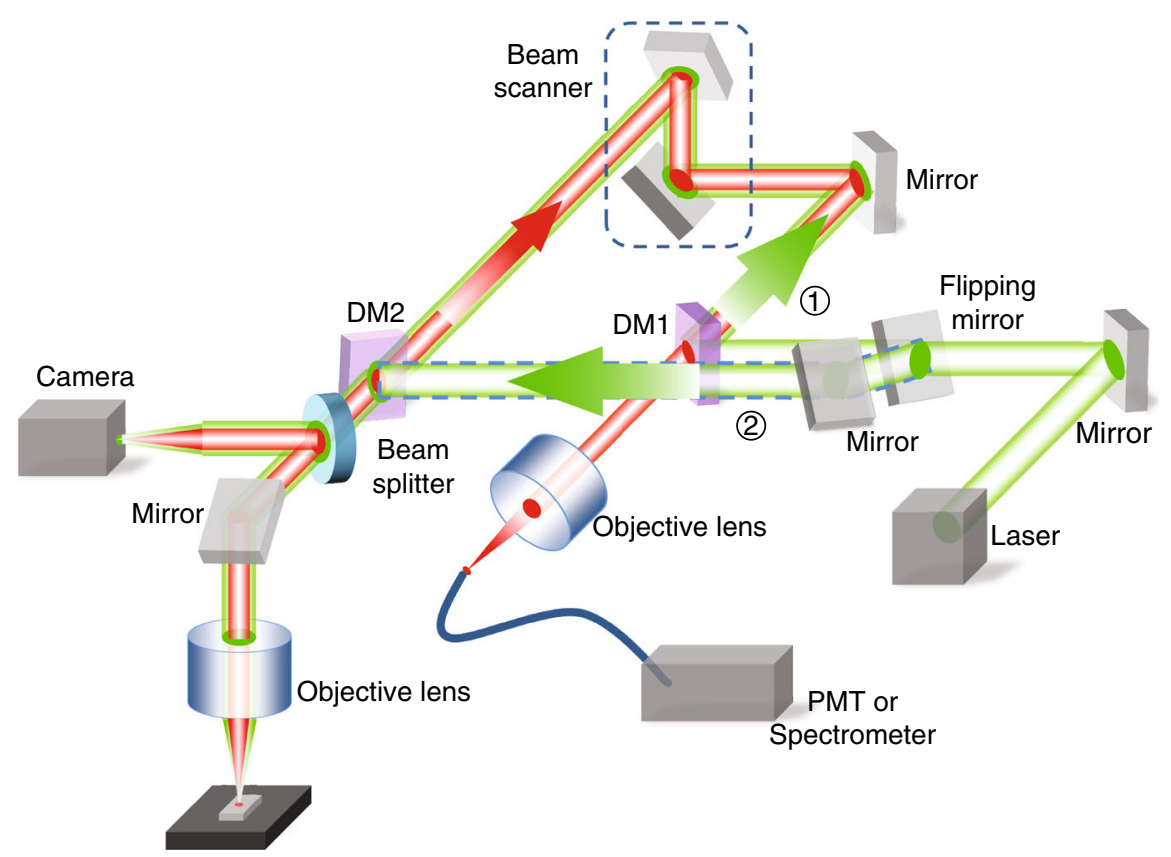

Fig. 4 Illustration of the optical measurement set-up. The optical path following the excitation laser is shown in green and that of the collected PL signal is shown in red. Flipping mirror switches the excitation beam to either (1) double scan mode or (2) single scan mode. DM1 and DM2: dichromatic mirrors

is fitted to an analytical expression that takes into account energy transport in a $2 \mathrm{D}$ plane (see Methods). The fitting yielded a propagation length of $43 \mu \mathrm{m}$. The remaining discrepancy between the experimentally extracted energy propagation decay length and the simulated one likely arises from the uncertainty in the oxide layer thickness $(2-4 \mathrm{~nm})$ and the sensitivity of the fitting to the noise in the PL intensity, especially for the first few data points.

\section{Discussion}

The current experiments on the hybrid MOS with unstructured Ag plates have not been designed to optimize the efficiency in the exciton-SPP-exciton conversion process, but instead to demonstrate the cascaded exciton transfer process mediated by SPP. Engineering local light-matter interaction via antennas, at the excitation or detection sites, might allow us to improve the energy conversion efficiency ${ }^{29}, 30$. However, such structures will introduce ambiguity if excitons are really involved in the excitation or detection step, since SPPs can be directly excited by laser or out-coupled via antennas. Similarly, the cascaded exciton energy transfer can only be demonstrated conceptually in TMD LHS. If only one TMD material is present, scattered SPPs rather than PL from excitons would dominate the out-coupled signal. In contrast, the energy down-conversion in LHS allows one to distinguish the exciton emission from the out-coupled SPPs at the collection site.

In conclusion, we have demonstrated that the energy of excitons, excited in one material region of a monolayer TMD LHS, can be transferred to another material region by coupling to the SPPs in an MOS hybrid material platform with the plasmonic substrate essentially acting as a planar waveguide. The planar MOS geometry used in our work has several advantages. First, the geometry is extremely simple, and no nanofabrication process is necessary to realize $2 \mathrm{D}$ exciton energy transfer. Second, the plasmonic substrate supporting a large optical density of states harvests the exciton emissions with high efficiency and enhances the emission intensity. Our experiments show that the atomically sharp interface in high-quality TMD LHS does not present as an energy transfer barrier. Combined with versatile methods recently developed to control light-matter interaction using plasmonic metasurfaces at the subdiffraction length scale, our work will stimulate further developments incorporating the newly created atomically thin semiconductor heterostructures for a wide range of applications including optical signal processing and photovoltaics.

\section{Methods}

Growth of TMD LHS. Two representative lateral $\mathrm{WS}_{2} / \mathrm{MoS}_{2}$ heterostructures were respectively obtained with the one-step synthesis using atmospheric pressure CVD (APCVD) and two-step synthesis by combining APCVD in the first growth step and low pressure CVD in the second step. Compositional alloying in the multi-elements CVD synthesis could be avoided with a low temperature CVD synthesis using perylene-3,4,9,10-tetracarboxylic acid tetrapotassium saltas the seeding promoter. The monolayer TMD LHS were synthesized at $650{ }^{\circ} \mathrm{C}$ for $5 \mathrm{~min}$ with a heating rate of $15^{\circ} \mathrm{C} / \mathrm{min}$ and a mixed $\mathrm{Ar} / \mathrm{H}_{2}$ gas flow $(15 / 1 \mathrm{sccm})$. In the one-step synthesis, homemade quartz reactors were used to precisely control the gas flow and the reactions of starting reactants in an one-inch tube furnace growth system.

Synthesis of colloidal Ag plates. The single-crystalline Ag plates were synthesized using a platinum $(\mathrm{Pt})$-nanoparticle-catalyzed and ammonium hydroxide $\left(\mathrm{NH}_{4} \mathrm{OH}\right)$-controlled polyol reduction method ${ }^{17}$. The synthesis was conducted at room temperature in a chemical solution.

TEM and STEM-HAADF. Field-emission transmission electron microscope (TEM, JEOL, operated at $200 \mathrm{kV}$ with a point-to-point resolution of $0.2 \mathrm{~nm}$ ) was used in this work. Monolayer LHS samples were transferred with a conventional polymethyl methacrylate-assisted technique to $\mathrm{Cu}$ grids for TEM and aberration corrected STEM high-angle annular dark-field (STEM-HAADF) imaging.

ALD of $\mathbf{A l}_{\mathbf{2}} \mathbf{O}_{\mathbf{3}}$ layer. $\mathrm{ALD}$ of high-quality $\mathrm{Al}_{2} \mathrm{O}_{3}$ was grown as a protection layer on the surfaces of $\mathrm{Ag}$ to avoid oxidation of $\mathrm{Ag}$ plate during the optical measurements. After the synthesis of $\mathrm{Ag}$ plate, the samples are immediately capped with a 3-nm-thick $\mathrm{Al}_{2} \mathrm{O}_{3}$ in the ALD chamber to preserve the sample quality.

Optical measurements. The PL measurements were performed with a home-built laser scanning confocal microscope system, as shown in Fig. 4. There are two 
operational modes (single-scan and double-scan modes), which can be switched by a flipping mirror. In the double-scan mode, both the laser excitation spot and PL collection location are scanned simultaneously, i.e., the excitation spot $(\sim 1 \mu \mathrm{m}$ in diameter) and PL are from the same region of the sample. In the single scan mode, the excitation laser is fixed at a specific location. The location from which the PL is collected is raster scanned across the sample, i.e., PL from locations different from the excitation spot is recorded. This single scan mode was used for all experiments reported here, aiming to demonstrate exciton energy transfer in the MOS structure.

Curve fitting. The decay of the PL intensity is fitted with the following equation:

$$
I=I_{\mathrm{b}}+I_{0} \frac{\mathrm{e}^{-x / \Lambda}}{x},
$$

where $I_{\mathrm{b}}$ is the background signal, $I_{0}$ represents the initial intensity at the launching site and $\Lambda$ is the propagation length (or decay length) associated with the energy transfer process. In addition to the exponential decay due to SPP loss, the $x^{-1}$ dependence arises from the fact that the SPPs propagate in all directions in a $2 \mathrm{D}$ plane. $\Lambda$ is found to be $\sim 43 \mu \mathrm{m}$ in our experiment. The experimental data shown in Fig. 3 is normalized to the PL intensity at the launching site:

$$
I=\frac{\int\left(I_{\text {sig }}-I_{\mathrm{bg}}\right)}{\int\left(I_{\text {launch }}-I_{\mathrm{bg}}\right)},
$$

where $I_{\text {sig }}, I_{\mathrm{bg}}$ and $I_{\text {launch }}$ are PL intensities at the collection site, background and PL intensity at the launching site, respectively. As seen from Fig. $2 \mathrm{f}, I_{\text {sig }}$ contains both the $\mathrm{MoS}_{2}$ exciton PL component and the out-coupled SPP. We collected PL from the same location while moving the excitation to ensure that the relative intensity of these two components (i.e., exciton PL and out-coupled SPP) remained the same for all measurements. Because of the fixed ratio between these two components, using the total output intensity to extract the energy transfer distance is equivalent to using the $\mathrm{MoS}_{2}$ PL intensity for this purpose.

Simulations. The exciton-SPP coupling and SPP characterization were numerically studied through full-wave simulations in COMSOL Multiphysics in the frequency domain using radio frequency module at $630 \mathrm{~nm}$. The structure was modeled as a 2D multilayer waveguide excited with an electric dipole positioned inside the $\mathrm{WS}_{2}$ layer. As shown in Fig. 3, the SPP mode generated at the Ag-dielectric-WS ${ }_{2}$ interface is significantly extended in air (with an exponential decay), lying close to the light line. Power distribution in the proximity of the excitation is illustrated in Fig. 3d. In order to avoid near-field effects close to the excitation point, power intensity is sampled with at least $20 \mu \mathrm{m}$ lateral distance from the excitation to calculate the propagation distance. In the $2 \mathrm{D}$ model, an exponential decay along the propagation direction was used to extract the propagation length, which is then used to produce red curve in Fig. 3b.

The $\mathrm{WS}_{2}$ layer is modeled as a sheet admittance ${ }^{31}$.

$$
Y=\frac{j k_{0} t\left(\varepsilon_{\mathrm{WS}_{2}}-1\right)}{\eta_{0}}
$$

where $Y$ is the admittance, $\varepsilon_{\mathrm{WS}_{2}}$ is the permittivity of the material and $t=0.81 \mathrm{~nm}$ is the layer thickness. $k_{0}$ and $\eta_{0}$ are free-space wavenumber and intrinsic impedance, respectively.

The simulation domain is a circular segment (Fig. $4 \mathrm{~b}$ ) truncated with a perfectly matched layer at the top, and a 400 -nm-thick grounded silver layer at the bottom. The thickness of the oxide layers on both sides of theWS $\mathrm{S}_{2}$ are estimated as $3 \mathrm{~nm}$ from fabrication process. Maximum mesh sizes of $5 \mathrm{~nm}$ and $15 \mathrm{~nm}$ are used for oxide layers and silver substrate, respectively. The air domain is meshed with maximum element size of $15 \mathrm{~nm}$ in the proximity of the $\mathrm{WS}_{2}$ and $80 \mathrm{~nm}$ in the far-field. The propagation distance is calculated from the SPP power sampled $100 \mathrm{~nm}$ above the silver interface.

Data availability. The data that support the findings of this study are available from the corresponding author upon reasonable request.

Received: 26 September 2016 Accepted: 28 April 2017

Published online: 26 June 2017

\section{References}

1. Zhang, X., Lin, C., Tseng, Y., Huang, K. \& Lee, Y. Synthesis of lateral heterostructures of semiconducting atomic layers. Nano. Lett. 15, 410-415 (2015).

2. Huang, C. et al. Lateral heterojunctions within monolayer $\mathrm{MoSe}_{2}-\mathrm{WSe}_{2}$ semiconductors. Nat. Mater. 13, 1096-1101 (2014).
3. Gong, Y. et al. Vertical and in-plane heterostructures from $\mathrm{WS}_{2} / \mathrm{MoS}_{2}$ monolayers. Nat. Mater. 13, 1135-1142 (2014).

4. Duan, X. et al. Lateral epitaxial growth of two-dimensional layered semiconductor heterojunctions. Nat. Nanotechnol. 9, 1024-1030 (2014).

5. Li, M. et al. Epitaxial growth of a monolayer $\mathrm{WSe}_{2}-\mathrm{MoS}_{2}$ lateral p-n junction with an atomically sharp interface. Science 349, 524-528 (2015).

6. Hill, H. M. et al. Observation of Excitonic Rydberg States in Monolayer $\mathrm{MoS}_{2}$ and $\mathrm{WS}_{2}$ by Photoluminescence Excitation Spectroscopy. Nano. Lett. 15, 2992-2997 (2015).

7. Eliseevaa, S. V. \& Bünzli, J.-C. G. Lanthanide luminescence for functional materials and bio-sciences. Chem. Soc. Rev. 39, 189-227 (2010).

8. Frischmann, P. D., Mahata, K. \& Würthner, F. Powering the future of molecular artificial photosynthesis with light-harvesting metallosupramolecular dye assemblies. Chem. Soc. Rev. 42, 1847 (2013).

9. Bao, J. \& Bawendi, M. G. A colloidal quantum dot spectrometer. Nature. 523, 67-73 (2015)

10. Cui, Q., Ceballos, F., Kumar, N. \& Zhao, H. Transient absorption microscopy of monolayer and bulk WSe ${ }_{2}$. ACS. Nano. 8, 2970-2976 (2014).

11. Palummo, M., Bernardi, M. \& Grossman, J. C. Exciton radiative lifetimes in two-dimensional transition metal dichalcogenides. Nano. Lett. 15, 2794-2800 (2015).

12. Moody, G. et al. Intrinsic homogeneous linewidth and broadening mechanisms of excitons in monolayer transition metal dichalcogenides. Nat. Commun. 6, 8315 (2015).

13. Poellmann, C. et al. Resonant internal quantum transitions and femtosecond radiative decay of excitons in monolayer $\mathrm{WSe}_{2}$. Nat. Mater. 14, 889-893 (2015).

14. Moody, G., Schaibley, J. \& Xu, X. Exciton dynamics in monolayer transition metal dichalcogenides. J. Opt. Soc. Am. B. 33, C39-C49 (2016).

15. Lee, C. et al. Atomically thin $\mathrm{p}-\mathrm{n}$ junctions with van der Waals heterointerfaces. Nat. Nanotechnol. 9, 676-681 (2014).

16. Hong, X. et al. Ultrafast charge transfer in atomically thin $\mathrm{MoS}_{2} / \mathrm{WS}_{2}$ heterostructures. Nat. Nanotechnol. 9, 682-686 (2014).

17. Wang, C. et al. Giant colloidal silver crystals for low-loss linear and nonlinear plasmonics. Nat. Commun. 6, 7734 (2015).

18. Liu, X. et al. Strong light-matter coupling in two-dimensional atomic crystals. Nat. Photonics 9, 30-34 (2015).

19. Pacifici, D., Lezec, H. J. \& Atwater, H. A. All-optical modulation by plasmonic excitation of CdSe quantum dots. Nat. Photonics 1, 402-406 (2007).

21. Wei, H., Ratchford, D., Li, X., Xu, H. \& Shih, C.-K. Propagating surface plasmon induced photon emission from quantum dots. Nano. Lett. 9, 4168-4171 (2009).

22. Goodfellow, K. M., Chakraborty, C., Beams, R., Novotny, L. \& Vamivakas, A. N. On-chip optical plasmon detection with an atomically thin semiconductor. Nano. Lett. 15, 5477-5481 (2015).

23. Goodfellow, K. M., Beams, R., Chakraborty, C., Novotny, L. \& Vamivakas, A. $\mathrm{N}$. Integrated nanophotonics based on nanowire plasmons and atomically thin material. Optica 1, 149-152 (2014).

24. Wang, H., Kalantar-Zadeh, K., Kis, A., Coleman, J. N. \& Strano, M. S. Electronics and optoelectronics of two-dimensional transition metal dichalcogenides. Nat. Nanotechnol. 7, 699-712 (2012).

25. Zeng, H. \& Cui, X. An optical spectroscopic study on two dimensional group-VI transition metal dichalcogenides. Chem. Soc. Rev. 44, 2629-2642 (2015).

26. Geim, A. K. \& Grigorieva, I. V. Van der Waals heterostructures. Nature. 499, 419-425 (2013).

27. Wu, Y. et al. Intrinsic optical properties and enhanced plasmonic response of epitaxial silver. Adv. Mater. 26, 6106-6110 (2014).

28. Liu, H. et al. Optical properties of monolayer transition metal dichalcogenides probed by spectroscopic ellipsometry. Appl. Phys. Lett. 105, 201905 (2014).

29. Kern, J. et al. Nanoantenna-enhanced light-matter interaction in atomically thin $\mathrm{WS}_{2}$. ACS Photonics 2, 1260-1265 (2015).

30. Sidiropoulos, T. P. H., Maier, S. A. \& Oulton, R. F. Efficient low dispersion compact plasmonic-photonic coupler. Opt. Express. 20, 12359-12365 (2012).

31. Vakil, A. \& Engheta, N. Transformation optics using graphene. Science 332, 1291-1294 (2011).

\section{Acknowledgements}

S.G. acknowledges research grants from the Ministry of Science and Technology (MOST) in Taiwan (MOST-104-2628-M-007-001, MOST-105-2112-M-007-011-MY3, and MOST-105-2633-M-007-003). Y.-H.L. acknowledges financial supports from the AOARD grant FA2386-16-1-4009 (co-funded with ONRG), the MOST grants (MOST-105-2112-M-007-032-MY3, MOST-105-2119-M-007-027, and MOST-105-2633 M-007-003), and the Academia Sinica Research Program on Nanoscience and Nanotechnology. J.S. was partially supported by the National Natural Science Foundation of China (Grant Nos. 11674032 and 11374037). The work at the UT-Austin was partially supported by NSF DMR-1306878, EFMA-1542747, the Welch Foundation (Grant Nos. F-1662 and F-1802), and NSF ECCS-0953311. The collaboration between National 
Tsing-Hua University and The University of Texas at Austin is facilitated by the Global Networking Talent 3.0 Program by the Ministry of Education in Taiwan.

\section{Author contributions}

S.G., X.L., Y.-H.L., C.-K.S., and A.A. designed and supervised the project. J.S. led the optical experiments with assistance from M.-H.L., H.-Y.C., Y.W., and C.-A.C. The TMD sample growth, transfer, and TEM imaging were performed by Y.-T.C., X.-Q.Z, and

C.-A.C. M.-H.L. synthesized Ag crystals. N.M.E performed the simulations. J.S., S.G., X. L., N.M.E., Y.-H.L., C.-K.S., and A.A. wrote the manuscript. All authors discussed the results and commented on the manuscript at all stages.

\section{Additional information}

Supplementary Information accompanies this paper at doi:10.1038/s41467-017-00048-y.

Competing interests: The authors declare no competing financial interests.

Reprints and permission information is available online at http://npg.nature.com/ reprintsandpermissions/
Publisher's note: Springer Nature remains neutral with regard to jurisdictional claims in published maps and institutional affiliations.

(c) (i) Open Access This article is licensed under a Creative Commons Attribution 4.0 International License, which permits use, sharing, adaptation, distribution and reproduction in any medium or format, as long as you give appropriate credit to the original author(s) and the source, provide a link to the Creative Commons license, and indicate if changes were made. The images or other third party material in this article are included in the article's Creative Commons license, unless indicated otherwise in a credit line to the material. If material is not included in the article's Creative Commons license and your intended use is not permitted by statutory regulation or exceeds the permitted use, you will need to obtain permission directly from the copyright holder. To view a copy of this license, visit http://creativecommons.org/ licenses/by/4.0/.

(C) The Author(s) 2017 